\title{
Measuring ex vivo drug susceptibility in Plasmodium vivax isolates from Cambodia
}

Suwanna Chaorattanakawee ${ }^{1,2^{*}} \mathbb{1 0}$, Chanthap Lon', Soklyda Chann ${ }^{1}$, Kheang Heng Thay ${ }^{3}$, Nareth Kong ${ }^{3}$, Yom You ${ }^{3}$, Siratchana Sundrakes ${ }^{1}$, Chatchadaporn Thamnurak', Sorayut Chattrakarn' ${ }^{1}$, Chantida Praditpol', Kritsanai Yingyuen ${ }^{1}$, Mariusz Wojnarski ${ }^{1}$, Rekol Huy ${ }^{3}$, Michele D. Spring ${ }^{1}$, Douglas S. Walsh' ${ }^{1}$, Jaymin C. Patel ${ }^{4}$, Jessica Lin ${ }^{4}$, Jonathan J. Juliano ${ }^{4}$, Charlotte A. Lanteri ${ }^{1}$ and David L. Saunders ${ }^{1,5}$

\begin{abstract}
Background: While intensive Plasmodium falciparum multidrug resistance surveillance continues in Cambodia, relatively little is known about Plasmodium vivax drug resistance in Cambodia or elsewhere. To investigate P. vivax antimalarial susceptibility in Cambodia, 76 fresh P. vivax isolates collected from Oddar Meanchey (northern Cambodia) in 2013-2015 were assessed for ex vivo drug susceptibility using the microscopy-based schizont maturation test (SMT) and a Plasmodium pan-species lactate dehydrogenase (pLDH) ELISA. P. vivax multidrug resistance gene 1 ( $p v m d r 1$ ) mutations, and copy number were analysed in a subset of isolates.

Results: Ex vivo testing was interpretable in $80 \%$ of isolates using the pLDH-ELISA, but only $25 \%$ with the SMT. Plasmodium vivax drug susceptibility by pLDH-ELISA was directly compared with 58 P. falciparum isolates collected from the same locations in 2013-4, tested by histidine-rich protein-2 ELISA. Median PLDH-ELISA IC 50 of $P$. vivax isolates was significantly lower for dihydroartemisinin (3.4 vs $6.3 \mathrm{nM}$ ), artesunate (3.2 vs $5.7 \mathrm{nM}$ ), and chloroquine (22.1 vs $103.8 \mathrm{nM}$ ) than P. falciparum but higher for mefloquine ( $92 \mathrm{vs} 66 \mathrm{nM}$ ). There were not significant differences for lumefantrine or doxycycline. Both P. vivax and $P$. falciparum had comparable median piperaquine $I C_{50}(106.5$ vs $123.8 \mathrm{nM})$, but some $P$. falciparum isolates were able to grow in much higher concentrations above the normal standard range used, attaining up to 100 -fold greater $\mathrm{IC}_{50} \mathrm{~S}$ than $P$. vivax. A high percentage of $P$. vivax isolates had pvmdr 1 Y $976 \mathrm{~F}(78 \%)$ and $\mathrm{F} 1076 \mathrm{~L}$ (83\%) mutations but none had pvmdr1 amplification.

Conclusion: The findings of high P. vivax $I C_{50}$ to mefloquine and piperaquine, but not chloroquine, suggest significant drug pressure from drugs used to treat multidrug resistant $P$. falciparum in Cambodia. Plasmodium vivax isolates are frequently exposed to mefloquine and piperaquine due to mixed infections and the long elimination half-life of these drugs. Difficulty distinguishing infection due to relapsing hypnozoites versus blood-stage recrudescence complicates clinical detection of $P$. vivax resistance, while well-validated molecular markers of chloroquine resistance remain elusive. The $\mathrm{pLDH}$ assay may be a useful adjunctive tool for monitoring for emerging drug resistance, though more thorough validation is needed. Given high grade clinical chloroquine resistance observed recently in neighbouring countries, low chloroquine $I C_{50}$ values seen here should not be interpreted as susceptibility in the absence of clinical data. Incorporating pLDH monitoring with therapeutic efficacy studies for individuals with $P$. vivax will help to further validate this field-expedient method.
\end{abstract}

Keywords: Drug resistance, Plasmodium vivax, Cambodia, Ex vivo assay, pvmdr1

\footnotetext{
*Correspondence: suwann67@yahoo.com; suwanna.cho@mahidol.ac.th

${ }^{2}$ Department of Parasitology and Entomology, Faculty of Public Health,

Mahidol University, Bangkok, Thailand

Full list of author information is available at the end of the article
} 


\section{Background}

Cambodia continues to be at the epicenter of globally emerging multidrug resistant malaria. This has prompted intensive elimination efforts accompanied by surveillance to characterize Plasmodium falciparum resistance. Over the past several decades, $P$. falciparum has developed resistance to numerous drugs, particularly in Southeast Asia where high grade treatment failures have been documented [1-4]. Since 2000, the use of artemisinin (ART)based combination therapy (ACT) as first-line treatment has been implemented in nearly all malaria endemic areas to overcome resistance developing as a result of monotherapy treatments. However, $P$. falciparum resistance to ART emerged in 2006, just a few years after introduction of artesunate-mefloquine (AS-MQ) in Cambodia [5, 6], and was later confirmed on a large scale by intensive multidisciplinary surveillance studies [7]. In 2013, the first high grade clinical failures of dihydroartemisininpiperaquine (DHA-PPQ) were reported [8]. DHA-PPQ had only recently been introduced as first-line therapy in Cambodia. Reduced in vitro $P$. falciparum susceptibility to PPQ developed on a background of artemisinin resistance here $[9,10]$, with specific molecular mechanisms elucidated soon thereafter $[11,12]$.

While intensive surveillance for $P$. falciparum continues in Cambodia, little is known about Plasmodium vivax drug resistance. Clinical $P$. vivax resistance is far more difficult to characterize due to difficulties distinguishing true recrudescence of resistant parasites, reinfections with new blood-stage $P$. vivax infections, and relapsing infections by liver stage hypnozoites. Moreover, inability to maintain long-term culture of $P$. vivax parasites prevents reproducible assessments of parasite drug susceptibilities. Plasmodium vivax resistance is generally assumed to be less pronounced than $P$. falciparum, as blood-stage $P$. vivax infection remains clinically susceptible to most available anti-malarials. CQ resistant $P$. vivax was first reported in 1989 from Papua New Guinea (PNG) and Papua Indonesia [13-16], where CQ monotherapy remains ineffective. Chloroquine-resistant vivax isolates in this region have been found to harbour polymorphisms in the $p v m d r 1$ ( $P$. vivax multidrug resistance 1) gene, whereas amplification of the gene has been associated with reduced susceptibility to MQ and other drugs in vitro [17-20]. Sporadic cases of CQ failure have since been reported in parts of Southeast Asia and South America [21-24], but there is no clear evidence that these are associated with $p v m d r 1$ mutations in these regions. Thus, the usefulness of this putative drug resistance marker for detecting emerging resistance remains uncertain.

Artemisinin-based combination therapy is now recommended for use as first-line agent for all malaria in areas of multidrug resistant $P$. falciparum, particularly where clinical failures have been documented [25, 26]. In Cambodia, DHA-PPQ was used as first-line therapy for both vivax and falciparum malaria since 2011 to respond to declining efficacy of CQ for P. vivax treatment in specific northern and western provinces where the clinical cure rate had dropped to $80-90 \%$, though cure rates have remained near $100 \%$ elsewhere [27-29]. The approach was also implemented to simplify drug administration and overcome diagnostic difficulties. Limited diagnostic capacity makes distinguishing $P$. vivax from $P$. falciparum microscopically challenging, despite overall increases in diagnostic capacity after years of effort. Frequent relapse of latent $P$. vivax following treatment for blood stage $P$. falciparum infections, and high prevalence of difficult to distinguish mixed species infections pose further challenges. While the use of DHA-PPQ to treat CQ-sensitive $P$. vivax is thought to be effective and convenient [26], it may in fact exacerbate resistance to both $P$. vivax and $P$. falciparum [30].

To investigate $P$. vivax anti-malarial susceptibility in Cambodia, fresh $P$. vivax isolates collected from Northern provinces from 2013 to 2015 were assessed for sensitivity to commonly used drugs in short-term culture. Growth inhibition was measured by both the microscopy-based schizont maturation test (SMT) and Plasmodium pan-species lactate dehydrogenase (pLDH) ELISA. SMT had been considered the conventional method for $P$. vivax drug testing, but not sensitive for low parasitaemia samples. It is also labour-intensive and interpretation of results is subjective. pLDH ELISA has emerged, offering notable advantages. pLDH has demonstrated higher sensitivity for detection in settings of low $P$. vivax growth rate and very low parasitaemia, improving yields for $\mathrm{IC}_{50}$ determination [31]. Results from these assays were then compared with those from concurrently collected $P$. falciparum isolates analysed in both the novel pLDH assay and the previously established HRP-2 assay [32]. Plasmodium vivax multidrug resistance gene 1 (pvmdr1) mutations and copy number, which have been proposed as candidate markers for drug resistance, were analyzed in a subset of isolates. This is the first report that we are aware of that documents ex vivo $P$. vivax drug susceptibility in Cambodia, and provides baseline data for future surveillance and elimination efforts.

\section{Methods}

\section{Study site, sample collection and processing}

Plasmodium isolates were obtained from volunteers with uncomplicated malaria enrolled in an anti-malarial drug resistance surveillance study conducted June 2013-October 2015, in Oddar Meanchey province, northern Cambodia. The study was approved by the 
Cambodian National Ethics Committee for Health Research (NECHR), and the Walter Reed Army Institute of Research (WRAIR) Institutional Review Board (protocol WR1576). All subjects were $\geq 13$ years old without a history of anti-malarial drug use within the past 7 days. Diagnosis of malaria was performed using Giemsa-stained peripheral blood smears, and confirmed later by real-time PCR [9]. A total of $76 P$. vivax isolates were collected from volunteers with mono $P$. vivax from June 2013 to October 2015, while 58 P. falciparum isolates were collected for comparison from those with mono P. falciparum infections from January to August 2014. After informed consent, patient venous blood samples were collected in sodium heparin and directly tested fresh for ex vivo drug susceptibility within $6 \mathrm{~h}$ of phlebotomy without blood centrifugation, leukocyte depletion or culture adaptation. Additional blood was collected in an EDTA tube for molecular marker analysis for $P$. vivax drug resistance.

In the first $29 P$. vivax isolates, a portion of heparinized blood was centrifuged, and cell pellets including buffy coat were passed through CF11 cellulose (What$\operatorname{man}^{\mathrm{TM}}$, Maidstone, UK) or Plasmodipur filters (EuroProxima, The Netherlands) to remove human white blood cells (WBC). WBC depleted samples were tested for drug susceptibility side by side with whole blood samples. Samples were treated with $45 \%$ Percoll in only the first $3 P$. vivax isolates to separate late stage parasites and enrich for and synchronize at early stages for the schizont maturation test (SMT) [33]. An overall schematic for the experiment is shown in Fig. 1.

\section{Preparation of dried drug plates}

Dried drug-coated plates were prepared using published methods, and tested against the $P$. falciparum W2 reference clone for quality control [32, 34]. All drugs were provided by Chemical Repository of the Walter Reed Army Institute of Research (WRAIR). Briefly, dihydroartemisinin (DHA), artesunate (AS), mefloquine hydrochloride (MQ), quinine sulfate hydrate $(\mathrm{QN})$, chloroquine diphosphate (CQ), and piperaquine phosphate (PPQ) were coated onto 96 well plates. Atovaquone (ATQ),

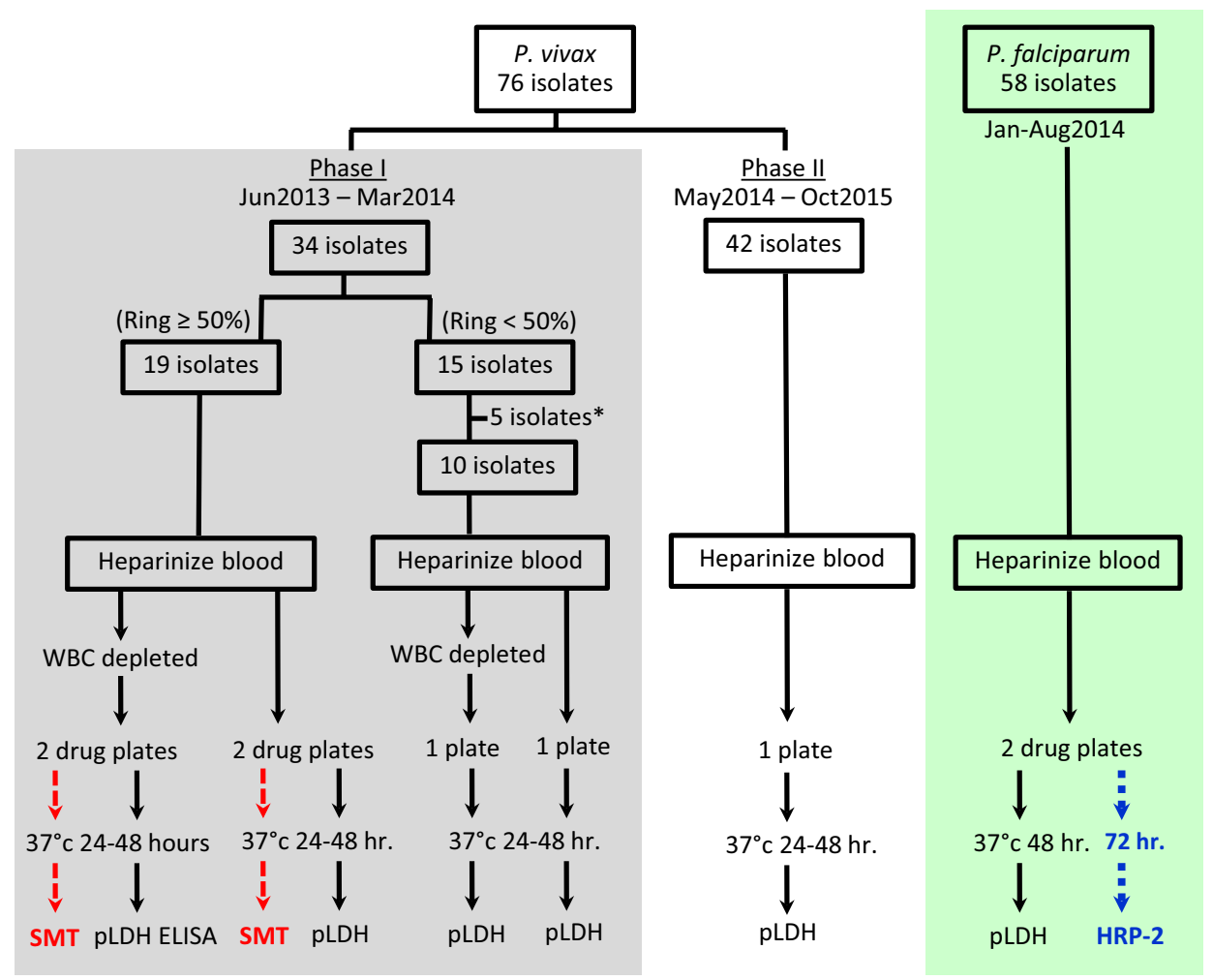

Fig. 1 Schematic for ex vivo experiments. Plasmodium vivax ex vivo experiments were divided into 2 phases. In Phase I, the schizont maturation test (SMT) was performed concurrently with the pLDH ELISA. Blood samples were processed to deplete white blood cells, and incubated with drugs of interest in parallel with whole blood samples. The SMT was conducted only in samples with $\geq 50 \%$ ring stages. $* 5$ isolates with ring stage $<50 \%$ in initial samples were not tested for drug susceptibility, but 3 of them had Percoll treatment applied to separate late stage parasites and enrich for early stages for the SMT. In phase II, only pLDH ELISA was performed on whole blood samples. For P. falciparum testing, pLDH ELISA was performed concurrently with the HRP-2 ELISA with $48 \mathrm{~h}$ incubation times used in the PLDH ELISA to ensure meaningful comparison of $P$. vivax susceptibility, while the previously established $72 \mathrm{~h}$ incubation was followed for the HRP-2 method 
lumefantrine (LUM), doxycycline (DOX) and artemisone (ATM) were added only to later plate lots once the initial methods had been established, and as a result were tested in only 76\% (ATQ and LUM), 60\% (DOX), and 49\% (ATM) of isolates, respectively. Final drug concentrations (after adding samples) ranged from 0.095 to $70 \mathrm{nM}$ for DHA, 0.07 to $52 \mathrm{nM}$ for AS, 0.66 to $482 \mathrm{ng} / \mathrm{ml}$ for MQ, 2.18 to $1596 \mathrm{nM}$ for QN, 5.31 to $3877 \mathrm{nM}$ for CQ, 0.9 to $674 \mathrm{nM}$ for PPQ, 0.38 to $273 \mathrm{nM}$ for ATQ, 0.13 to $95 \mathrm{nM}$ for LUM, 285 to $207,943 \mathrm{nM}$ for DOX, and 0.02 to $25 \mathrm{ng} /$ $\mathrm{ml}$ for ATM. Starting in 2013, some P. falciparum isolates were able to grow in extremely high PPQ concentrations [35]. From 2014 onward, increased PPQ concentration (3.4-53,905 $\mathrm{nM})$ was used in addition to the standard dilutions to ensure accurate inhibitory concentrations could be determined. The top row of each plate served as a drug-free control.

\section{Immediate ex vivo drug susceptibility assay}

At the time of diagnosis (before treatment), fresh Plasmodium isolates were tested for sensitivity to anti-malarials by culturing on dried drugs coated plates and measuring growth inhibition. For the $P$. vivax assay, blood samples were adjusted to $2 \%$ haematocrit in McCoy's $5 \mathrm{~A}$ medium supplemented with $20 \% \mathrm{AB}$ blood-group serum, and added to dried drug coated plates. Plates were then incubated at $37^{\circ} \mathrm{C}$ in a candle jar for $24-48 \mathrm{~h}$, and parasite maturation in drug-free wells was checked by microscopy at $24 \mathrm{~h}$ and then every $6 \mathrm{~h}$ until $48 \mathrm{~h}$. Parasite growth inhibition over the established concentration ranges was measured by the microscopy-based schizont maturation test (SMT) using the modified WHO microtest $[36,37]$ for the first $19 P$. vivax isolates with $>50 \%$ early stage parasites in initial samples and Plasmodium pan-species lactate dehydrogenase (pLDH) ELISA [31, 38] for all isolates, side by side (Fig. 1). For the SMT, incubation was stopped when $\geq 40 \%$ of parasites in drug-free wells had reached the schizont stage. Thick smear examination of samples from each well was performed, and the number of schizonts per 200 asexual stage parasites for each drug concentration was determined. The number of schizonts were plotted against drug concentrations, and $\mathrm{IC}_{50} \mathrm{~s}$ were estimated by nonlinear regression analysis using GraphPad Prism version 6.0. For the pLDH-ELISA assay, incubation was stopped when $\geq 40 \%$ schizont stages were reached in drug-free wells, or at $48 \mathrm{~h}$ if parasite cultures did not reach at least $40 \%$ schizonts. Plates were frozen and later thawed for analysis of growth inhibition using the pLDH-ELISA. pLDH optical density (OD) readings were plotted against drug concentrations, and $\mathrm{IC}_{50} \mathrm{~s}$ were estimated as described above. Samples with poor growth rates, defined as $<40 \%$ schizonts in drug-free wells or a pLDH-OD ratio $<1.7$ between no-drug control wells and maximum tested drug concentrations, were excluded from data analysis for SMT or pLDH-ELISA, respectively. A "successful" $\mathrm{IC}_{50}$ assay result was defined as achieving a sigmoidal concentration-response when testing serial drug dilutions for at least one of the tested drugs.

For the $P$. falciparum assay, the pLDH-ELISA [38] and the established histidine-rich protein-2 (HRP-2) ELISA [32] was used to simultaneously test field isolates and reference laboratory clones (W2 and 3D7). Culture conditions used in the $P$. falciparum assay were adjusted to $1.5 \%$ haematocrit in 0.5\% Albumax RPMI 1640. Parasite growth inhibition was assessed by pLDH-ELISA and HRP-2 ELISA after 48 and $72 \mathrm{~h}$ incubation, respectively. The same incubation period $(48 \mathrm{~h})$ for $P$. vivax and $P$. falciparum were used in the pLDH ELISA to ensure meaningful comparison of susceptibility results. The previously established $72 \mathrm{~h}$ incubation was followed for the HRP-2 method. $\mathrm{IC}_{50} \mathrm{~s}$ were estimated as described above. Isolates with reduced susceptibility to PPQ capable of growing in maximum drug concentrations could not be interpolated using the standard PPQ dilution range (0.9$674 \mathrm{nM})$. To accurately estimate $\mathrm{IC}_{50}$ dose-response, the curve was replotted by fitting 'zero-growth' HRP2 or pLDH OD values at the extrapolated PPQ concentration of 53,905 nM [35, 39].

\section{Plasmodium vivax multi-drug resistance (pvmdr1) SNPs and copy number analysis}

Genomic DNA was prepared from EDTA-anticoagulated blood using the QIAamp DNA Mini Kit (Qiagen) and used to analyse pvmdr1 Y976F and F1076L mutations and copy number variation. Multiplex real-time PCR was used to assess $p v m d r 1$ copy number. The $p v m d r 1$ (target) and $p v$ aldolase (reference) genes were both amplified by RT-PCR, following methods described by Lin et al. [40]. Plasmids containing cloned fragments of $p v m d r 1$ and $p v$ aldolase, developed using the TOPO XL PCR Cloning Kit (Invitrogen, Carlsbad, CA), were used as positive controls in each experiment. Reactions were performed in duplicate. Threshold cycle $(\mathrm{Ct})$ values were used to calculate the relative quantitation of pvmdr1 copy number by the Pfaffl method [41]. Copy number was determined by rounding to the nearest integer and was considered increased if $>1$. For pvmdr1 Y976F and F1076L analysis, PCR was performed to amplify a $647 \mathrm{bp}$ region of the $p v m d r 1$ gene covering these SNPs, using the conditions previously described in Lin et al. and amplified products were directly sequenced [40].

\section{Statistical analysis}

Statistical analysis was performed using Graph-Pad Prism version 6.0 (GraphPad Software, Inc, San Diego, CA, USA). Parasite drug susceptibilities were expressed 
as median $\mathrm{IC}_{50} \mathrm{~s}$ for all isolates. Differences in susceptibility between groups were determined using non-parametric Mann-Whitney or Kruskal-Wallis tests. Comparison of $\mathrm{IC}_{50} \mathrm{~s}$ attained from pLDH and HRP-2 ELISA were made using Wilcoxon matched pair testing. Assay correlation was evaluated using Spearman's correlation test. Spearman test was also used to examine the correlation between $\mathrm{IC}_{50}$ and ratio of early/mature stage parasites (EM ratio) at start of assay to determine the effect of initial $P$. vivax stage on LDH-ELISA $\mathrm{IC}_{50}$ results.

\section{Results}

\section{Ex vivo $P$. vivax assay development using the schizont maturation test}

An overall schematic for the experiment is shown in Fig. 1. Of $76 P$. vivax isolates collected from Oddar Meanchey Province between June 2013 and October 2015, 71 fresh isolates were tested for ex vivo sensitivity to anti-malarials. Parasite density ranged from 2500 to $38,000 \mu \mathrm{L}^{-1}$ with variation in the initial parasite stage. Synchronous $(\geq 80 \%)$ early and mature trophozoite stages were found in 13 and 19 samples, respectively, while the rest included mixed stages. In an effort to improve suitability for the schizont maturation test (SMT) by synchronizing isolates at the early stage, the first 3 isolates were treated with $45 \%$ Percoll to separate late stage parasites and enrich for early stage. Although Percoll treatment helped to increase a proportion of early stages in samples, trophozoites remained, leading to failure to reach $80 \%$ early stage for all 3 samples. One explanation is that the density of $45 \%$ Percoll normally can separate the schizonts and very late trophozoites, leaving earlier trophozoites and ring stages in the samples. In addition, perfect separation efficiency of Percoll density centrifugation method may not be achievable in practice as even if synchronized late stages are found in interphase layer, mixed stage parasites may still be found in the pellet. The effect of white blood cell (WBC) depletion processes on parasite maturation were compared for the first 29 isolates, but far fewer schizonts in drug-free wells were observed in WBC-depleted samples $(0-29 \%$ schizonts), compared to whole blood samples $(0-72 \%$ schizont). None of the WBC depleted samples reached the threshold for successful culture for SMT ( $\geq 40 \%$ schizonts in drug-free wells) [37]. Given that neither Percoll treatment nor WBC depletion improved assay conditions, both were abandoned. To examine the effects of WBC on the assay, the pLDH assay was run on whole blood and packed RBC specimens of these 29 P. vivax isolates, side by side. Good correlations were found for all drugs tested ( $\rho=0.5-0.9, P<0.05)$, though slightly reduced $\mathrm{IC}_{50} \mathrm{~s}$ were found after removing WBC for DHA, AS, and CQ with median difference of $=-0.6$,
$-0.8,-6.2$, respectively. No significant differences were found for other drugs. Reasonable correlation of results attained from both approaches suggested assay reliability when using whole blood samples.

In ex vivo assays of whole blood samples, erythrocytic P. vivax maturation was observed for all but 5 isolates in no-drug control wells over $48 \mathrm{~h}$ culture resulting in $2-72 \%$ schizonts (median $=21 \%$ ). However, only 22 of 71 isolates were able to develop to $\geq 40 \%$ schizonts. The time to maximum ex vivo schizont growth varied from 24 to $48 \mathrm{~h}$, with a median of $48 \mathrm{~h}$, though it tended to be earlier for cultures with mature trophozoite stages taking only $24-30 \mathrm{~h}$. There was a positive correlation between higher ratios of early/mature trophozoites (EM ratio) and time taken to reach maximum schizont growth (Spearman $\rho=0.63 ; P<0.001$ ). Of the first $19 P$. vivax isolates with $>50 \%$ early stage parasites in initial samples, SMT yielded an interpretable $\mathrm{IC}_{50}$ in only 6 isolates (32\% success rate). Since the SMT was found to have fewer interpretable results and was significantly more labourintensive than pLDH-ELISA, the SMT was not performed in the remaining experiments.

\section{Comparability of ex vivo Plasmodium falciparum susceptibility in the HRP-2 and pLDH ELISA assays}

Given the challenges of the SMT, the PLDH ELISA was evaluated next. In order to first establish the relevance of the pLDH ELISA assay for comparing $P$. falciparum to $P$. vivax resistance profiles, $P$. falciparum $\mathrm{IC}_{50}$ results in our routinely used HRP-2 assay and the pLDH ELISA were compared. Figure 2 revealed similarities between $P$. falciparum isolate $\mathrm{IC}_{50}$ in the HRP-2 and pLDH ELISA assays for chloroquine (CQ), lumefantrine (LUM), the artemisinins (ARTs), piperaquine (PPQ), and doxycycline (DOX). Wilcoxon pairwise $\mathrm{IC}_{50}$ comparisons were significantly higher in the pLDH assay for mefloquine (MQ). Figure 3 illustrates global correlation for $P$. falciparum isolates susceptibility between the 2 assays. There were moderate to strong correlations between results obtained for $P$. falciparum isolates in the pLDH assay and HRP-2 ELISAs for all drugs tested, with the exception of DOX. The latter findings were despite similarities in median values overall.

\section{Comparison of ex vivo $P$. vivax and $P$. falciparum drug susceptibility in Cambodia 2013-2015 using the pLDH-ELISA method}

pLDH-ELISA $\mathrm{IC}_{50} \mathrm{~s}$ to anti-malarials of Cambodian $P$. vivax isolates collected during 2013-2015 are shown in Fig. 4. Limited SMT data as described above hindered meaningful comparison of results from these 2 assays. pLDH-ELISA $\mathrm{IC}_{50}$ results from P. falciparum isolates collected in the same area, and from laboratory clones are 


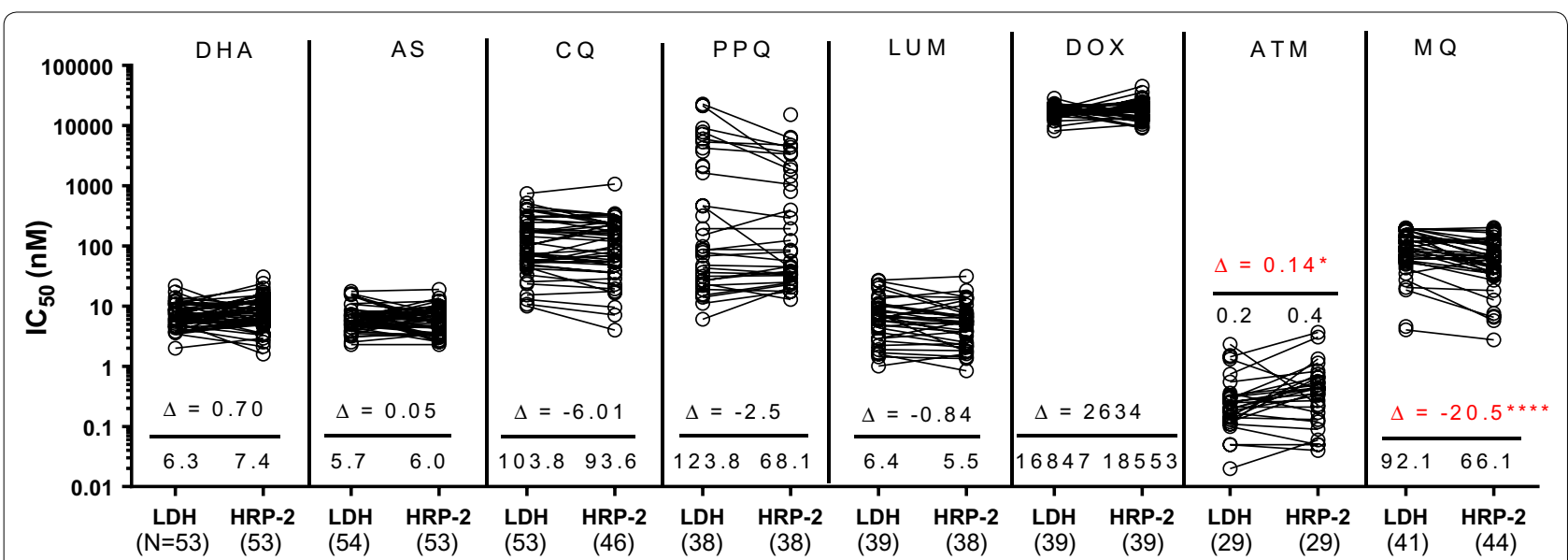

Fig. 2 Comparison of $P$. falciparum $I C_{50}$ values attained from $\mathrm{PLDH}$ and HRP-2 ELISA. Fresh $P$. falciparum isolates were tested for drug susceptibility using pLDH and HRP-2 ELISA, side by side. Median differences $(\Delta)$ are indicated below each pairwise comparison with values from the respective assays for each isolate joined by black lines. Unconnected dots are those where a corresponding value could not be obtained in either the pLDH or HRP-2 ELISA. Median $I C_{50}$ and numbers of evaluable isolates from the respective assays appear above the $X$-axis, Significant $P$-values from the Wilcoxon pair test are indicated as ${ }^{*}(P<0.05),{ }^{* *}(P<0.01),{ }^{* *}(P<0.001),{ }^{* * *}(P<0.0001)$

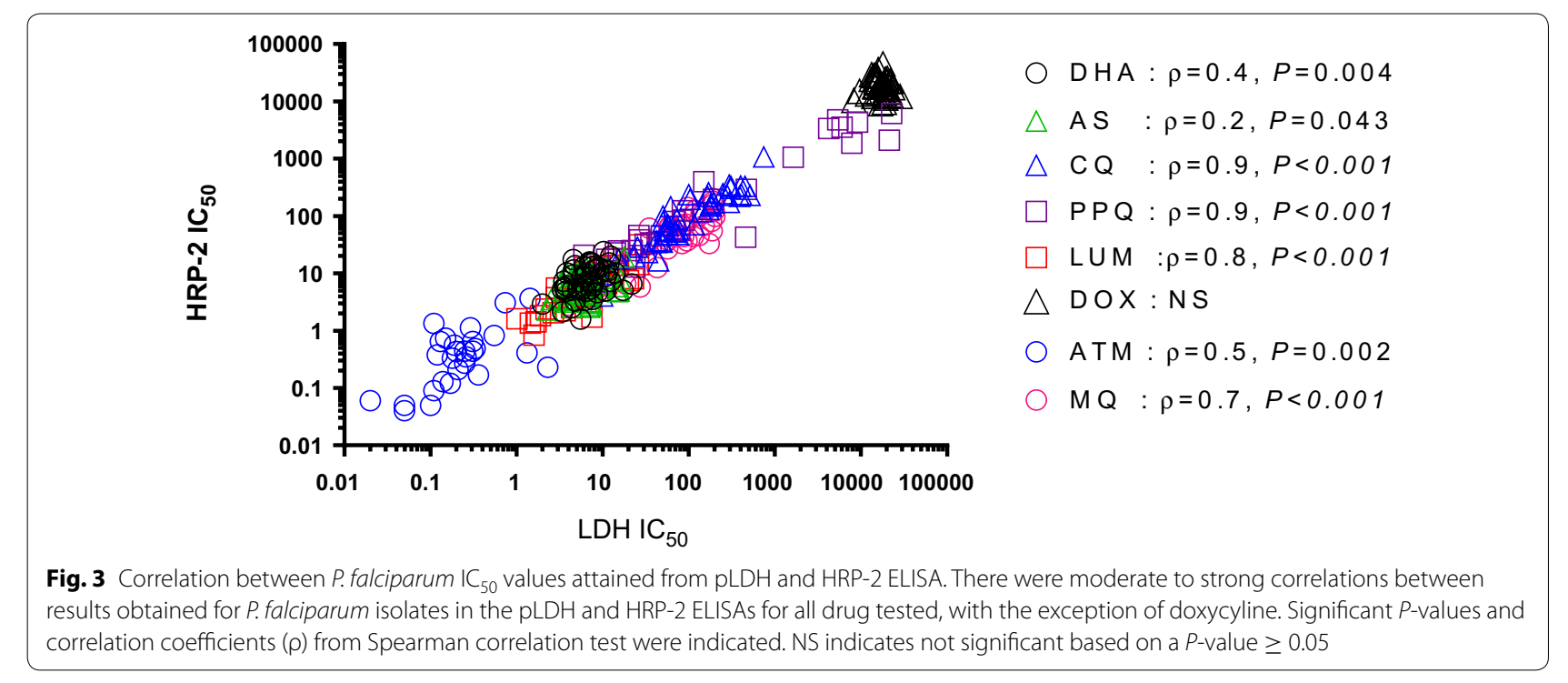

also presented in Fig. 4 for comparison. $\mathrm{IC}_{50}$ results of P. falciparum laboratory clones were able to successfully discriminate expected susceptibility profiles for the 3D7 (CQ-sensitive, MQ-resistant) and W2 strains (CQ-resistant, MQ-sensitive). pLDH-ELISA $\mathrm{IC}_{50}$ s were significantly lower for $P$. vivax than $P$. falciparum clinical isolates for dihydroartemisinin (DHA), artesunate (AS), and CQ, while no differences were found for LUM where both had low $\mathrm{IC}_{50}$ or DOX where both had high $\mathrm{IC}_{50}$ (Fig. 4). In contrast, pLDH-ELISA $\mathrm{IC}_{50} \mathrm{~s}$ of $P$. vivax isolates were significantly higher for artemisone (ATM) and MQ than $P$. falciparum in this assay.
Both $P$. vivax and $P$. falciparum had comparable median PPQ $\mathrm{IC}_{50}$ (106.5 vs $123.8 \mathrm{nM}$ respectively), but some $P$. falciparum isolates were able to grow in much higher concentrations above the normal standard curve used (Fig. 4). Although a statistically significant difference was not detected initially when comparing PPQ susceptibility between $P$. vivax and $P$. falciparum based on the pLDH assay, $67 \%$ of $P$. falciparum isolates (39 out of 58 total) were found to survive exposure to maximum standard curve PPQ levels $(674 \mathrm{nM})$. Some isolates were found to have few 100 -fold greater $\mathrm{IC}_{50} \mathrm{~s}(470-22,000 \mathrm{nM})$ using a new testing paradigm with increased maximal 


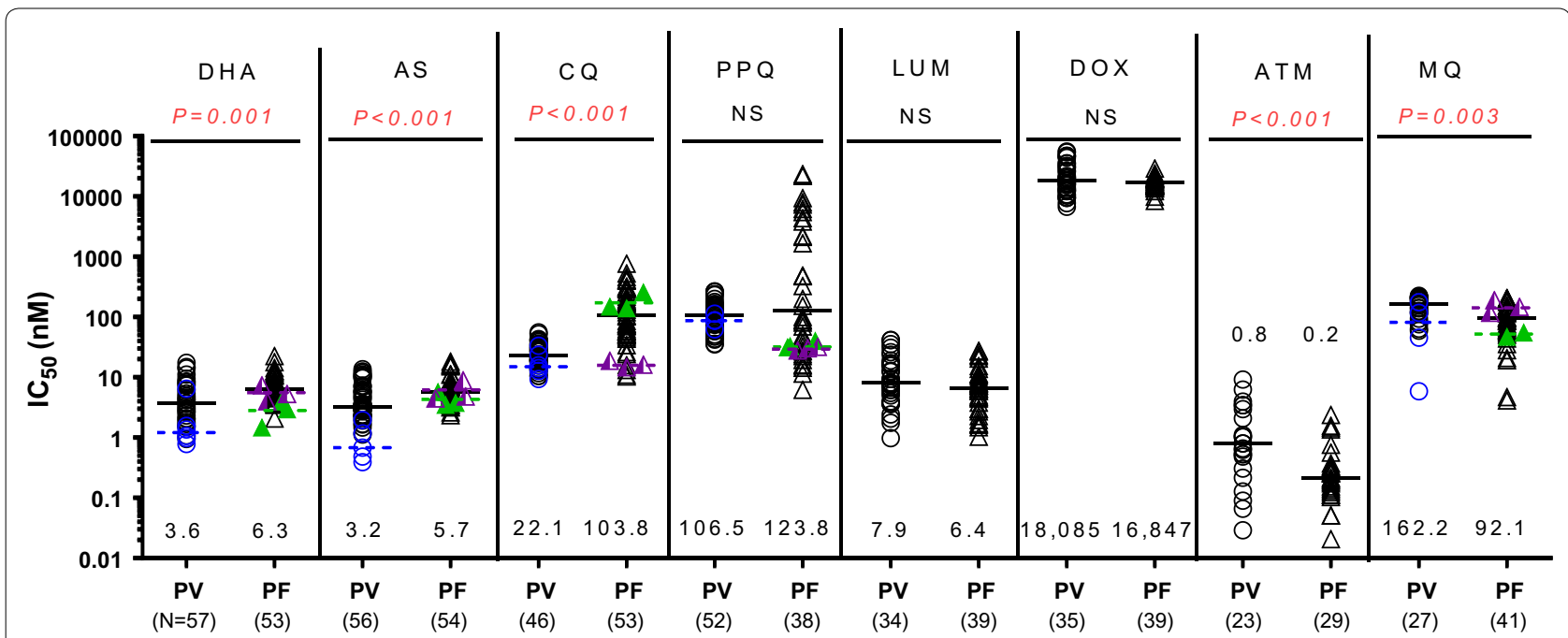

Fig. 4 Ex vivo drug susceptibility of $P$. vivax and $P$. falciparum isolates collected from Oddar Meanchey Province (northern Cambodia) during 2013-2015. pLDH-ELISA IC ${ }_{50}$ of fresh P. vivax (PV) and P. falciparum (PF) isolates against commonly used anti-malarials are presented as black dot plots with black median bars, while SMT results for the only six evaluable PV isolates are indicated in blue. Circle represent PV isolates, while triangles represent PF. Median PLDH-ELISA IC ${ }_{50}$ and numbers of evaluable isolates appear above the X-axis, and the $P$-values for the Mann-Whitney test comparing $\mathrm{IC}_{50}$ S of $P$. vivax and $P$. falciparum appear at the top of the graph. NS indicates not significant based on a $P$-value $\geq 0.05$. pLDH-ELISA IC 50 of W2 (green) and 3D7 (purple) reference clones obtained from three independent assays are presented in scatter dot plot with bars representing averages

PPQ concentrations of $53,905 \mathrm{nM}$, compared to those obtained in previous years $(<50 \mathrm{nM})$. In contrast, none of the evaluated $P$. vivax isolates were able to grow in the highest tested PPQ concentration of $674 \mathrm{nM}$, suggesting greater overall susceptibility compared to $P$. falciparum. $P$. vivax susceptibility to PPQ increased over the period 2013-2015, while there were minor fluctuations or no change in parasite susceptibility to the other drugs tested (Additional file 1).

In contrast to the SMT, pLDH-ELISA was able to determine $\mathrm{IC}_{50}$ in $83 \%$ of $P$. vivax isolates (58/70) evaluated, though success rate was drug dependent, ranging from 66 to $83 \%$ for most drugs. There was < $20 \%$ success in achieving sigmoidal $\mathrm{IC}_{50}$ curves for quinine $(\mathrm{QN})$ and atovaquone (ATQ). High background optical density (OD) levels in maximally concentrated wells interfered with interpretation. An OD ratio $<1.7$ between drug-free control and maximum concentration wells precluded interpretable concentration-response curves. Therefore, QN and ATQ $\mathrm{IC}_{50}$ results were excluded from the analysis. Likewise MQ had a lower success rate (39\%) compared to other drugs. The high pLDH-OD background interference observed during QN, ATQ, and MQ testing was also observed in P. falciparum assays but had less effect on interpretability, and was not detected when $P$. falciparum was tested in the HRP-2 ELISA. The success rate of pLDH-ELISA for P. falciparum isolates ranged from 62 to $98 \%$ for all drugs tested.
Effect of initial $P$. vivax stage, assay incubation time and ex vivo growth efficiency on $\mathrm{pLDH}-\mathrm{ELISA}_{\mathrm{IC}} \mathrm{C}_{\mathbf{5 0}}$ results

To determine the effect of initial $P$. vivax stage on pLDH-ELISA results, the relationship between initial parasite stage and $\mathrm{IC}_{50}$ was examined (Table 1 ). There was a negative correlation between early/mature trophozoite $(\mathrm{EM})$ ratio and $\mathrm{IC}_{50}$ for $\mathrm{DOX}(\rho=-0.7)$ with lesser effects observed for DHA, AS and LUM $(\rho=-0.3$ to -0.5$)$. Three to four-fold greater $\mathrm{IC}_{50}$ for these drugs were detected in $P$. vivax isolates with synchronous mature trophozoites initially (EM ratio $\leq 0.25$ ) than those with synchronous early stages (EM ratio $\geq 4)$. However, there was only a borderline correlation between $\mathrm{EM}$ ratio and $\mathrm{IC}_{50}$ for $\mathrm{MQ}$, and no effect for other drugs.

The influence of assay incubation time on pLDHELISA $\mathrm{IC}_{50}$ results was examined, and there were noticeable effects when testing DHA, AS, LUM, and DOX. There were significant increases in $\mathrm{IC}_{50}$ for these drugs in isolates requiring shorter incubations (24-30 h) compared to longer ones (42-48 h) (Kruskall-Wallis $P$ value $<0.001-0.016)$. To assess the effect of ex vivo growth efficiency on assay results, pLDH-ELISA $\mathrm{IC}_{50}$ of isolates in the drug-free control assay reaching $\geq 40 \%$ schizonts (the designated threshold for SMT culture success) was compared with results of isolates having less growth efficiency. There were no differences in $\mathrm{IC}_{50}$ between the 2 groups for any of the drugs tested (Table 2). 
Table 1 Effect of initial $P$. vivax stage on pLDH-ELISA IC ${ }_{50}$ results

\begin{tabular}{|c|c|c|c|c|c|c|c|c|}
\hline \multirow[t]{3}{*}{ Drug } & \multirow{2}{*}{\multicolumn{3}{|c|}{$\begin{array}{l}\text { Spearman correlation test } \\
\text { for } \mathrm{IC}_{50} \text { and } \mathrm{EM} \text { ratio }\end{array}$}} & \multicolumn{4}{|c|}{ Mann-Whitney $U$ test } & \multirow[t]{3}{*}{$P$ value } \\
\hline & & & & \multicolumn{2}{|c|}{$E M$ ratio $\geq 4$} & \multicolumn{2}{|c|}{ EM ratio $\leq 0.25$} & \\
\hline & $\mathbf{N}$ & coefficient & $P$-value & $\mathrm{N}$ & median IC ${ }_{50}$ & $\mathbf{N}$ & median $\mathrm{IC}_{50}$ & \\
\hline $\mathrm{DHA}$ & 57 & $-0.34^{*}$ & $0.009^{*}$ & 11 & 2.6 & 12 & $7.5^{*}$ & $0.008^{*}$ \\
\hline AS & 56 & $-0.46^{*}$ & $<0.001^{*}$ & 11 & 2.9 & 11 & $9.4^{*}$ & $<0.001^{*}$ \\
\hline CQ & 46 & -0.12 & 0.445 & 12 & 23.8 & 4 & 28.1 & 0.716 \\
\hline PPQ & 52 & -0.01 & 0.922 & 12 & 104.3 & 7 & 99.5 & 0.612 \\
\hline LUM & 34 & $-0.42^{*}$ & $0.014^{*}$ & 4 & 5.6 & 7 & $25.4^{*}$ & $0.023^{*}$ \\
\hline DOX & 35 & $-0.70^{*}$ & $<0.001^{*}$ & 5 & 12,680 & 9 & $42,667^{*}$ & $0.003^{*}$ \\
\hline ATM & 23 & -0.12 & 0.593 & 3 & 0.61 & 7 & 2.0 & 0.137 \\
\hline MQ & 27 & -0.39 & $0.046^{*}$ & 4 & 161.2 & 4 & 203.7 & 0.083 \\
\hline
\end{tabular}

* Statistical significance

Table 2 There was little effect of ex vivo $P$. vivax growth on pLDH-ELISA IC ${ }_{50}$ results

\begin{tabular}{|c|c|c|c|c|c|}
\hline \multirow[t]{3}{*}{ Drug } & \multicolumn{4}{|c|}{ Maximum number of schizonts } & \multirow{3}{*}{$\begin{array}{l}P \text { value of Mann- } \\
\text { Whitney } U \text { test }\end{array}$} \\
\hline & \multicolumn{2}{|c|}{$<40 \%$ schizont } & \multicolumn{2}{|c|}{$\geq 40 \%$ schizont } & \\
\hline & $\mathrm{N}$ & median $I C_{50}$ & $\mathrm{~N}$ & median IC 50 & \\
\hline DHA & 37 & 3.0 & 20 & 4.1 & 0.108 \\
\hline AS & 37 & 3.0 & 19 & 3.5 & 0.166 \\
\hline CQ & 32 & 22.1 & 14 & 22.1 & 0.981 \\
\hline PPQ & 35 & 108.0 & 17 & 105.0 & 0.513 \\
\hline LUM & 23 & 7.3 & 11 & 9.3 & 0.118 \\
\hline DOX & 24 & 16,021 & 11 & 31,326 & 0.102 \\
\hline ATM & 15 & 0.6 & 8 & 1.0 & 0.175 \\
\hline MQ & 19 & 150.0 & 8 & 179.1 & 0.426 \\
\hline
\end{tabular}

\section{Plasmodium vivax drug resistance markers}

Plasmodium vivax multi-drug resistance (pvmdr1) Y976F and F1076L mutations and copy number were analysed for 23 P. vivax isolates collected in 2013. Of 23 evaluated isolates, $p v m d r 1 \mathrm{Y} 976 \mathrm{~F}$ and F1076L mutations were found in $78 \%(17 / 23)$ and $83 \%(19 / 23)$, respectively, composing $74 \%(17 / 23)$ double mutants, 9\% (2/23) single F1076L mutants, and 17\% (4/23) wild type isolates. None had pvmdr1 amplification.

\section{Discussion}

This is the first report of ex vivo $P$. vivax drug susceptibility testing of field isolates in Cambodia, providing important baseline data for ongoing resistance surveillance. The focus of containment and elimination efforts in Cambodia to date has been multidrug resistant $P$. falciparum malaria. Relatively little is known about $P$. vivax drug sensitivity due to the inability to culture $P$. vivax long term. The pLDH method bypasses this critical limitation by using fresh isolates, making it potentially practical and informative for measuring ex vivo $P$. vivax resistance. While further validation of the assay is needed, the correlations observed with the $\mathrm{IC}_{50}$ results with $P$. falciparum, in both the pLDH and previously established HRP-2 assays, lend support to its utility as a surveillance tool [32]. The P. falciparum HRP-2 assay has been carefully standardized in an effort to reliably produce interpretable $\mathrm{IC}_{50}$ results in $P$. falciparum field isolates over time [32]. However, it cannot be used in $P$. vivax which does not produce HRP-2. Unfortunately, we were unable to establish the $P$. vivax SMT using previously described methods. Potential explanations for the poor success rate of SMT could include low parasitaemia and drug residue in samples. Nearly $40 \%$ of $P$. vivax samples has parasitaemia $<0.1 \%$, and issues of self-medication and unregulated anti-malarial distribution in Cambodia are well documented [42]. Further, WBC depletion process seems to retard $P$. vivax growth leading to failure for SMT. The loss of $P$. vivax and time spent during the WBC filtration process could be possible reasons. However, less effect was observed in pLDH ELISA in which $\mathrm{IC}_{50}$ from whole blood, and WBC depleted samples testing were well correlated. This corresponded with the previous finding on P. falciparum HRP-2 method, suggesting the reliability of assay on whole blood sample without WBC removing [43]. Overall, in addition to being a field expedient method, the pLDH method was able to reveal some important information about $P$. vivax susceptibility.

With the possible exception of chloroquine, blood stage P. vivax is generally thought to have remained susceptible to a wide variety of anti-malarials, though data is limited. Using the pLDH-ELISA, $P$. vivax appeared significantly more susceptible to dihydroartemisinin (DHA), artesunate (AS), and chloroquine (CQ) than P. falciparum,but less susceptible to mefloquine (MQ), and artemisone (ATM), and similarly susceptible to lumefantrine (LUM), 
piperaquine (PPQ) and doxycycline (DOX). It should be noted that although DOX $\mathrm{IC}_{50}$ values were in the micromolar range, they were still below previously proposed values for resistance [44]. The present study reveals Cambodian $P$. vivax isolates appear to remain sensitive to CQ while resistance to other anti-malarials may be worse than previously assumed, though the absence of baseline values precludes definitive conclusions. Although previously established, well-controlled pLDH methods were used here to test both $P$. vivax and $P$. falciparum, inter-species difference and assay bias between 2 species cannot be ruled out. Inter-species comparisons require careful interpretation, especially in the absence of baseline data. In addition, mixed parasite stages found in $P$. vivax samples may confound pLDH results for drugs with stage-specific activity. It is possible that the $\mathrm{pLDH}$ results of $P$. vivax here represent an average overall susceptibility of mixed parasite stages. As an example, our findings indicated that mixed stage $P$. vivax remained susceptible to $C Q$, though $C Q$ has specific ring stage activity [37].

The present study brings to light important methodologic considerations for assessing $P$. vivax resistance in vitro. The effect of parasite growth efficiency on $P$. vivax $\mathrm{pLDH}$ assay was minimal. There were not significant differences in pLDH-ELISA $\mathrm{IC}_{50}$ for most drugs tested between isolates reaching $\geq 40 \%$ schizonts and those with less growth. Thus, the pLDH assay had utility even in $P$. vivax isolates failing to reach the $40 \%$ schizont target required for the SMT. pLDH-ELISA validity was also confirmed by successful discrimination of known susceptibility profiles for 3D7 (CQ sensitive, MQ-resistant) and W2 (CQ-resistant, MQ-sensitive) P. falciparum laboratory strains. When testing $P$. falciparum clinical isolates, pLDH-ELISA was able to detect PPQ resistance at several 100 -fold higher $\mathrm{IC}_{50}$, corresponding with results from the $72 \mathrm{~h}$ HRP-2 ELISA. Comparison with these previously well benchmarked assays further supports use of pLDH-ELISA for P. vivax isolate drug susceptibility testing.

Based on our pLDH-ELISA results, stage-specific drug activity on $P$. vivax growth was apparent for DOX and AS, but less pronounced for DHA, LUM and MQ (Table 1). Isolates initially at the trophozoite stage had significantly higher $\mathrm{IC}_{50} \mathrm{~s}$ to these drugs than those initially at the ring stage. Specific activity of chloroquine on ring stages, previously described for the schizont maturation test (SMT), was not detected here using the pLDH-ELISA. Comparative $P$. vivax testing at ring and trophozoite stages for the same isolates may confirm stage-specific activity of these drugs. Duration of drug incubation in the SMT is another factor previously reported to influence in vitro drug responses for P. vivax [37]. A prior statistical modelling study of SMT dose-response data indicated that only assays with initial ring stage parasitaemia $\geq 65 \%$ and a duration $\geq 35 \mathrm{~h}$ produce robust $\mathrm{IC}_{50}$ values [45]. More data is required to identify the threshold where the association between $\mathrm{IC}_{50}$ assay duration and parasite stage composition disappears in the pLDH ELISA.

Comparing findings of the present study to those reported previously for $P$. falciparum and $P$. vivax isolates from the Brazilian Amazon [46, 47] and Indonesia $[36,48,49]$ using pLDH ELISA and SMT assays, Cambodian isolates were found to be less susceptible to MQ and PPQ. However, Cambodian and Brazillian P. vivax isolates were more sensitive to CQ than in Papua Indonesia where $P$. vivax $\mathrm{CQ}$ resistance has emerged. Another ex vivo study reported $P$. vivax CQ resistance in $60 \%$ of isolates collected from the Thai-Myanmar border, and higher median $\mathrm{IC}_{50}$ than in Cambodia [50]. Although, comparability of the SMT and pLDH ELISA have yet to be formally established, these regional differences are not surprising. Reduced MQ and PPQ susceptibility of Cambodian isolates reflects higher drug pressure in the region from long term use. High grade PPQ resistance recently emerged in $P$. falciparum with resultant effects on sympatric $P$. vivax infection [10, 30]. Chloroquine resistant $P$. vivax infections have been detected in Indonesia since the 1990s and, in 2008, the national treatment guidelines for $P$. vivax were changed to ACT [51]. Approximately $60 \%$ of $P$. vivax patients treated with chloroquine experienced a recurrence within 28 days in studies from Malaysia and Vietnam $[24,52]$. Yet chloroquine sensitivity was better preserved in studies conducted in Cambodia [29, 44], the Brazilian Amazon [53], Myanmar [54], India [55] and Ethiopia [56].

Piperaquine phosphate $\mathrm{IC}_{50} \mathrm{~s}$ of Cambodian $P$. vivax isolates were higher than Indonesia, but the median was similar to those of $P$. falciparum from the same region. However, some Cambodian P. falciparum isolates were able to grow in much higher PPQ concentrations with up to 100 -fold greater $\mathrm{IC}_{50} \mathrm{~s}$ than $P$. vivax, suggesting greater overall susceptibility in $P$. vivax compared to $P$. falciparum. In 2012, AS-MQ was replaced with DHA-PPQ as national first-line treatment for both $P$. falciparum and $P$. vivax [57]. Since reduced ex vivo $P$. falciparum $\mathrm{PPQ}$ sensitivity of Cambodian isolates has corresponded with high grade failure of DHA-PPQ treatment in this region $[10,35]$, it raises a concern that DHA-PPQ may also become less effective for $P$. vivax. Unlike $P$. falciparum, P. vivax does not exhibit concomitant artemisinin resistance. In the absence of an established baseline value, it is possible that $P$. vivax has higher intrinsic PPQ $\mathrm{IC}_{50}$ in the assay than $P$. falciparum. Further, the decline observed in $P$. vivax $\mathrm{IC}_{50}$ after PPQ was introduced in 2013 may have been the result of a decline in overall parasite fitness in response to developing mutations, similar to 
previous observations in P. falciparum [58]. Regardless, clinical correlations are necessary to define the impact of increased PPQ $\mathrm{IC}_{50} \mathrm{~s}$ for P. vivax. Also to be explored is whether the change in treatment policy for $P$. vivax may have relieved chloroquine pressure. However, ongoing $P$. falciparum chloroquine resistance comparable to previously observed levels $[9,34]$ argues against this. Resistance in both parasite species would have been expected to subside if exposure had truly been reduced [59]. Definitive demonstration of assay utility will require comparison of ex vivo pLDH-ELISA results with clinical response in well-controlled $P$. vivax field studies. Limited ability to distinguish recrudescence, relapse, and reinfection clinically may confound interpretation.

While molecular data in the present study was limited by convenience sampling, and available resources, it does offer a few useful observations. The mechanism of CQ resistance in $P$. vivax remains unclear although a few studies have suggested associations with $P$. vivax multi-drug resistance gene (pvmdr1) Y976F mutation and pvcrt-o expression [17, 60]. In northern Cambodian isolates, $p v m d r 1$ Y976F mutants were observed at extremely high frequency yet when tested in the ex vivo assay, the isolates are CQ-sensitive. Accordingly, CQ has retained clinical efficacy as a $P$. vivax rescue agent in trials conducted there over the past several years by the USAMD-AFRIMS [29]. This argues against the usefulness of the Y976F mutation as a CQ resistance marker [17]. No pvmdr1 amplification was detected in Cambodian isolates, precluding correlation with ex vivo drug susceptibility to chloroquine or other anti-malarials. No attempt to measure stage-specific pvcrt-o expression in these clinical isolates was made here. Comparing our findings to those reported previously from other regions revealed geographical differences in $p v m d r 1$ amplification and mutation prevalence. The prevalence of Y976F and F1076L mutations were high in Cambodia, Indonesia, and Papua New Guinea (70-100\%) [36, 61, 62], but no mutant was detected in Brazil $[47,60]$. Moderate rates of mutation were previously found in Thailand (18-23\% for Y976F and 53-61\% for F1076L) [50, 62]. Corresponding to previous findings [40], most Cambodian isolates were double mutants $(74 \%)$, with single F1076L mutants found in $<10 \%$. The prevalence here differed compared to Thailand where a single F1076L mutation was seen in $>60 \%$. No pvmdr1 amplification was detected in northern Cambodian isolates tested in the present study, despite reduced susceptibility to $\mathrm{MQ}$. It is possible that if more sensitive assays targeting the pvmdr1 breakpoint were used [63], minority clones with pvmdr1 amplification may have been detected, given the polyclonal nature of vivax infections in this region [64-66]. Pvmdr1 amplification rates of 4-37\% have been observed in other regions of the country [30, 40]. Similarly, a 7-39\% amplification rate was reported in Thailand $[40,62]$. There was no pvmdr1 amplification in Indonesia [36, 67] and low prevalence in Brazil $(0.9-4 \%)$ [67, 68]. The lack or low rate of pvmdr1 amplification in some areas of Thailand and Cambodia with intense MQ pressure does not support previous evidence associating $p v m d r 1$ amplification with MQ pressure [67].

Although the pLDH-ELISA $\mathrm{IC}_{50}$ assay as described here represents an important first step to assess $P$. vivax drug susceptibility, the following caveats must be considered. The confounding factors of mechanism and speed of drug action must be taken into account when interpreting results obtained using different in vitro methods. High optical density (OD) background interference in wells containing maximal concentrations of some drugs tested caused low growth ratios and failure to achieve sigmoidal $\mathrm{IC}_{50}$ curves. This phenomenon was reported previously to depend on the nature of the drug being tested and detection methodology. Relatively higher OD background tends to be observed in non-artemisinin drugs with a more pronounced effect in ELISA-based assays $[69,70]$. This may relate to the later onset of action of non-artemisinins in the parasite life cycle, allowing ring stage parasites to continue to produce the proteins being assayed despite high drug concentrations. This may explain paradoxical growth seen at high concentrations of non-artemisinins common in the pLDH-ELISA, but less frequently in the HRP2-ELISA, and not at all in the microscopy-based SMT [69, 70]. This corresponds with the observation of parasite growth in high concentrations of atovaquone, mefloquine, and quinine in the pLDHELISA assay for both $P$. falciparum and $P$. vivax, but not in the HRP2 ELISA or SMT. The SMT's relatively low throughput, and challenges interpreting concentrationresponse curves for parasite cultures unable to develop to the threshold of $\geq 40 \%$ schizonts may limit its usefulness. The assay requires extensive experience on stage differentiation by operators to be effective and replicable. However, as the malaria map continues to shrink in Cambodia, it may be possible to better concentrate the expert microscopy skills needed to adequately perform the SMT in areas of greatest need.

\section{Conclusion}

Overall, the pLDH-ELISA has the potential to be a useful and replicable method to assess ex vivo $P$. vivax resistance in field isolates in Cambodia with reasonable throughput. Results correlated well with those observed for $P$. falciparum in both the $\mathrm{pLDH}$ and better established HRP-2 assays. While current malaria elimination 
efforts are focused on resistant $P$. falciparum malaria, $P$. vivax malaria may ultimately prove more difficult to eliminate due to its ease of transmission, dormancy, and only primaquine being effective at preventing $P$. vivax relapse. Current tools are also inadequate to differentiate relapse and recrudescence from re-infection. Evaluation of ex vivo $P$. vivax susceptibility using field isolates will likely prove a useful part of the armamentarium to monitor for growing resistance and predict risk of treatment failures. Clinical $P$. vivax resistance can be difficult to detect due to difficulty distinguishing relapsing disease from recrudescence and/or reinfection, and the pLDH assay may prove a useful adjunct in this regard. Incorporating ex vivo pLDH monitoring with therapeutic efficacy studies for individuals with $P$. vivax is advised to further validate this field-expedient method.

\section{Additional file}

Additional file 1. Ex vivo drug susceptibility of $P$. vivax by years.

\begin{abstract}
Abbreviations
DHA: dihydroartemisinin; AS: artesunate; MQ: mefloquine hydrochloride; QN: quinine sulfate hydrate; $C Q$ : chloroquine diphosphate; PPQ: piperaquine phosphate; ATQ: atovaquone; LUM: lumefantrine; DOX: doxycycline; ATM: artemisone; ATRs: artemisinins; ACT: artemisinin-based combination therapy; HRP-2: histidine-rich protein 2; pLDH: Plasmodium pan-species lactate dehydrogenase; ELISA: enzyme-linked immunosorbent assay; SMT: schizont maturation test; $I C_{50}: 50 \%$ inhibitory concentration; PCR: polymerase chain reaction; OD: optical density; Pvmdr1: Plasmodium vivax multi-drug resistance 1; Ct: threshold cycle; WHO: World Health Organization.
\end{abstract}

\section{Authors' contributions}

Study design Cambodia: SC, CL, DS, and CL; data collection: all; data analysis and interpretation: SC, DS, SS, CL, CL, JJ, JL; wrote manuscript: SC, DS, and CL. All authors read and approved the final manuscript.

\section{Author details}

${ }^{1}$ Department of Immunology and Medicine, Armed Forces Research Institute of Medical Science, Bangkok, Thailand. ${ }^{2}$ Department of Parasitology and Entomology, Faculty of Public Health, Mahidol University, Bangkok, Thailand. ${ }^{3}$ National Center for Parasitology, Entomology and Malaria Control, Phnom Penh, Cambodia. ${ }^{4}$ Division of Infectious Diseases, School of Medicine, University of North Carolina, Chapel Hill, NC, USA. ${ }^{5}$ US Army Medical Materiel Development Activity, Fort Detrick, Frederick, MD, USA.

\section{Acknowledgements}

We are grateful to members of the AFRIMS Immunology and Medicine Department in Thailand and Cambodia not listed as coauthors for their intrepid technical support. We thank Mr. William Ellis at the Walter Reed Army Institute of Research (WRAIR), and the Medicines for Malaria Venture for providing reference drug supplies.

\section{Competing interests}

The authors declare that they have no competing interests.

\section{Availability of data and materials}

The datasets generated and/or analysed during the current study are available from the corresponding author on reasonable request.

\section{Consent for publication}

All study subjects agreed to publication of the study results in the medical literature without their identity at consent prior to participation.

\section{Disclaimer}

The views expressed in this article are those of the authors and do not reflect the official policy of the US Department of the Army, US Department of Defense, or the US or Cambodian Governments.

\section{Ethics approval and consent to participate}

All protocols were approved by the Walter Reed Army Institute of Research (WRAIR) and the Cambodian National Ethics Committee for Health Research (NECHR). Protocol approval reference numbers include WR1576 (minimal risk). All study subjects provided informed consent prior to participation.

\section{Funding}

Funding for the study was provided by the US Armed Forces Health Surveillance Center and the National Institutes of Health, National Institutes of Allergy and Infectious Disease (Grant K08Al110651 to JTL and R21Al111108 to $J J J)$. The funding sources did not participate in data analysis or the final decision to publish the manuscript.

\section{Publisher's Note}

Springer Nature remains neutral with regard to jurisdictional claims in published maps and institutional affiliations.

Received: 5 June 2017 Accepted: 19 September 2017

Published online: 30 September 2017

\section{References}

1. Wongsrichanalai C, Pickard AL, Wernsdorfer WH, Meshnick SR. Epidemiology of drug-resistant malaria. Lancet Infect Dis. 2002;2:209-18.

2. Wongsrichanalai C, Sirichaisinthop J, Karwacki JJ, Congpuong K, Miller RS, Pang L, et al. Drug resistant malaria on the Thai-Myanmar and Thai-Cambodian borders. Southeast Asian J Trop Med Public Health. 2001:32:41-9.

3. Wernsdorfer WH, Payne D. The dynamics of drug resistance in Plasmodium falciparum. Pharmacol Ther. 1991;50:95-121.

4. Bjorkman A, Phillips-Howard PA. The epidemiology of drug-resistant malaria. Trans R Soc Trop Med Hyg. 1990;84:177-80.

5. Dondorp AM, Nosten F, Yi P, Das D, Phyo AP, Tarning J, et al. Artemisinin resistance in Plasmodium falciparum malaria. N Engl J Med. 2009:361:455-67.

6. Noedl H, Se Y, Schaecher K, Smith BL, Socheat D, Fukuda MM, Artemisinin Resistance in Cambodia 1 Study Consortium. Evidence of artemisininresistant malaria in western Cambodia. N Engl J Med. 2008;359:2619-20.

7. Takala-Harrison S, Clark TG, Jacob CG, Cummings MP, Miotto O, Dondorp AM, et al. Genetic loci associated with delayed clearance of Plasmodium falciparum following artemisinin treatment in Southeast Asia. Proc Natl Acad Sci USA. 2013;1 10:240-5.

8. Saunders DL, Vanachayangkul P, Lon C, U.S. Army Military Malaria Research Program, National Center for Parasitology Entomology and Malaria Control, Royal Cambodian Armed Forces. Dihydroartemisininpiperaquine failure in Cambodia. N Engl J Med. 2014;371:484-5.

9. Chaorattanakawee S, Saunders DL, Sea D, Chanarat N, Yingyuen K, Sundrakes S, et al. Ex Vivo drug susceptibility testing and molecular profiling of clinical Plasmodium falciparum isolates from Cambodia from 2008 to 2013 suggest emerging piperaquine resistance. Antimicrob Agents Chemother. 2015;59:4631-43.

10. Spring MD, Lin JT, Manning JE, Vanachayangkul P, Somethy S, Bun R, et al. Dihydroartemisinin-piperaquine failure associated with a triple mutant including kelch13 C580Y in Cambodia: an observational cohort study. Lancet Infect Dis. 2015;15:683-91.

11. Amaratunga C, Lim P, Suon S, Sreng S, Mao S, Sopha C, et al. Dihydroartemisinin-piperaquine resistance in Plasmodium falciparum malaria in Cambodia: a multisite prospective cohort study. Lancet Infect Dis. 2016;16:357-65.

12. Duru V, Khim N, Leang R, Kim S, Domergue A, Kloeung N, et al. Plasmodium falciparum dihydroartemisinin-piperaquine failures in Cambodia are associated with mutant $\mathrm{K} 13$ parasites presenting high survival rates in novel piperaquine in vitro assays: retrospective and prospective investigations. BMC Med. 2015;13:305. 
13. Baird JK, Basri H, Purnomo Bangs MJ, Subianto B, Patchen LC, et al. Resistance to chloroquine by Plasmodium vivax in Irian Jaya, Indonesia. Am J Trop Med Hyg. 1991;44:547-52.

14. Baird JK, Sustriayu Nalim MF, Basri H, Masbar S, Leksana B, Tjitra E, et al. Survey of resistance to chloroquine by Plasmodium vivax in Indonesia. Trans R Soc Trop Med Hyg. 1996;90:409-11.

15. Murphy GS, Basri H, Purnomo Andersen EM, Bangs MJ, Mount DL, et al. Vivax malaria resistant to treatment and prophylaxis with chloroquine. Lancet. 1993;341:96-100.

16. Rieckmann KH, Davis DR, Hutton DC. Plasmodium vivax resistance to chloroquine? Lancet. 1989;2:1183-4

17. Suwanarusk R, Russell B, Chavchich M, Chalfein F, Kenangalem E, Kosaisavee $V$, et al. Chloroquine resistant Plasmodium vivax: in vitro characterisation and association with molecular polymorphisms. PLoS ONE. 2007:2:e1089.

18. Marfurt J, de Monbrison F, Brega S, Barbollat L, Muller I, Sie A, et al. Molecular markers of in vivo Plasmodium vivax resistance to amodiaquine plus sulfadoxine-pyrimethamine: mutations in pvdhfr and pvmdr1. J Infect Dis. 2008;198:409-17.

19. Suwanarusk R, Chavchich M, Russell B, Jaidee A, Chalfein F, Barends M, et al. Amplification of pvmdr 1 associated with multidrug-resistant Plasmodium vivax. J Infect Dis. 2008;198:1558-64.

20. Imwong M, Pukrittayakamee S, Pongtavornpinyo W, Nakeesathit S, Nair S, Newton $P$, et al. Gene amplification of the multidrug resistance 1 gene of Plasmodium vivax isolates from Thailand, Laos, and Myanmar. Antimicrob Agents Chemother. 2008;52:2657-9.

21. Marlar T, Myat Phone K, Aye YuS, Khaing Khaing G, Ma S, Myint O. Development of resistance to chloroquine by Plasmodium vivax in Myanmar. Trans R Soc Trop Med Hyg. 1995:89:307-8.

22. Soto J, Toledo J, Gutierrez P, Luzz M, Llinas N, Cedeno N, et al. Plasmodium vivax clinically resistant to chloroquine in Colombia. Am J Trop Med Hyg. 2001;65:90-3.

23. Phillips EJ, Keystone JS, Kain KC. Failure of combined chloroquine and high-dose primaquine therapy for Plasmodium vivax malaria acquired in Guyana, South America. Clin Infect Dis. 1996;23:1171-3.

24. Thanh PV, Hong NV, Van NV, Louisa M, Baird K, Xa NX, et al. Confirmed Plasmodium vivax resistance to chloroquine in Central Vietnam. Antimicrob Agents Chemother. 2015;59:7411-9.

25. World Health Organization. Guidelines for the treatment of malaria. 3rd ed. Geneva: World Health Organization; 2015.

26. Gogtay N, Kannan S, Thatte UM, Olliaro PL, Sinclair D. Artemisinin-based combination therapy for treating uncomplicated Plasmodium vivax malaria. Cochrane Database Syst Rev. 2013;10:CD008492.

27. Poravuth $Y$, Socheat D, Rueangweerayut $R$, Uthaisin C, Pyae Phyo A, Valecha $\mathrm{N}$, et al. Pyronaridine-artesunate versus chloroquine in patients with acute Plasmodium vivax malaria: a randomized, double-blind, noninferiority trial. PLOS ONE. 2011;6:e14501.

28. Leang R, Barrette A, Bouth DM, Menard D, Abdur R, Duong S, et al. Efficacy of dihydroartemisinin-piperaquine for treatment of uncomplicated Plasmodium falciparum and Plasmodium vivax in Cambodia, 2008 to 2010. Antimicrob Agents Chemother. 2013;57:818-26.

29. Amaratunga C, Sreng S, Mao S, Tullo GS, Anderson JM, Chuor CM, et al. Chloroquine remains effective for treating Plasmodium vivax malaria in Pursat province, Western Cambodia. Antimicrob Agents Chemother. 2014;58:6270-2.

30. Khim N, Andrianaranjaka V, Popovici J, Kim S, Ratsimbasoa A, Benedet C, Barnadas $C$, et al. Effects of mefloquine use on Plasmodium vivax multidrug resistance. Emerg Infect Dis. 2014;20:1637-44.

31. Druilhe P, Brasseur P, Blanc C, Makler M. Improved assessment of Plasmodium vivax response to antimalarial drugs by a colorimetric double-site Plasmodium lactate dehydrogenase antigen capture enzyme-linked immunosorbent assay. Antimicrob Agents Chemother. 2007;51:2112-6.

32. Rutvisuttinunt $W$, Chaorattanakawee $S$, Tyner SD, Teja-Isavadharm $P$, Se Y, Yingyuen $\mathrm{K}$, et al. Optimizing the HRP-2 in vitro malaria drug susceptibility assay using a reference clone to improve comparisons of Plasmodium falciparum field isolates. Malar J. 2012;11:325

33. Russell B, Suwanarusk R, Malleret B, Costa FT, Snounou G, Baird JK, et al. Human ex vivo studies on asexual Plasmodium vivax: the best way forward. Int J Parasitol. 2012;42:1063-70.

34. Tyner SD, Lon C, Se Y, Bethell D, Socheat D, Noedl H, et al. Ex vivo drug sensitivity profiles of Plasmodium falciparum field isolates from Cambodia and Thailand, 2005 to 2010, determined by a histidine-rich protein-2 assay. Malar J. 2012;1 1:198.

35. Chaorattanakawee S, Lon C, Jongsakul K, Gawee J, Sok S, Sundrakes S, et al. Ex vivo piperaquine resistance developed rapidly in Plasmodium falciparum isolates in northern Cambodia compared to Thailand. Malar J. 2016;15:519.

36. Marfurt J, Chalfein F, Prayoga P, Wabiser F, Wirjanata G, Sebayang B, et al. Comparative ex vivo activity of novel endoperoxides in multidrug-resistant Plasmodium falciparum and P. vivax. Antimicrob Agents Chemother. 2012;56:5258-63.

37. Russell B, Chalfein F, Prasetyorini B, Kenangalem E, Piera K, Suwanarusk R, et al. Determinants of in vitro drug susceptibility testing of Plasmodium vivax. Antimicrob Agents Chemother. 2008;52:1040-5.

38. Druilhe P, Moreno A, Blanc C, Brasseur PH, Jacquier P. A colorimetric in vitro drug sensitivity assay for Plasmodium falciparum based on a highly sensitive double-site lactate dehydrogenase antigencapture enzyme-linked immunosorbent assay. Am J Trop Med Hyg. 2001:64:233-41.

39. Saunders DL, Chaorattanakawee S, Gosi P, Lanteri C, Somethy S, Kuntawunginn W, et al. Atovaquone-proguanil remains a potential stopgap therapy for multidrug-resistant Plasmodium falciparum in areas along the Thai-Cambodian border. Antimicrob Agents Chemother. 2015;60:1896-8.

40. Lin JT, Patel JC, Kharabora O, Sattabongkot J, Muth S, Ubalee R, et al. Plasmodium vivax isolates from Cambodia and Thailand show high genetic complexity and distinct patterns of $P$. vivax multidrug resistance gene 1 (pvmdr1) polymorphisms. Am J Trop Med Hyg. 2013;88:1116-23.

41. Pfaffl MW. A new mathematical model for relative quantification in realtime RT-PCR. Nucleic Acids Res. 2001;29:e45.

42. Bourdier F. Health inequalities, public sector involvement and malaria control in Cambodia. Sojourn. 2016;31:81-115.

43. Chaorattanakawee S, Lanteri CA, Sundrakes S, Yingyuen K, Gosi P, Chanarat $\mathrm{N}$, et al. Attenuation of Plasmodium falciparum in vitro drug resistance phenotype following culture adaptation compared to fresh clinical isolates in Cambodia. Malar J. 2015;14:486.

44. Javelle E, Madamet M, Gaillard T, Velut G, Surcouf C, Michel R, et al. Delayed Onset of Plasmodium falciparum malaria after doxycycline prophylaxis in a soldier returning from the Central African Republic. Antimicrob Agents Chemother. 2016;60:2592-3.

45. Kerlin DH, Boyce K, Marfurt J, Simpson JA, Kenangalem E, Cheng Q, et al. An analytical method for assessing stage-specific drug activity in Plasmodium vivax malaria: implications for ex vivo drug susceptibility testing. PLoS Negl Trop Dis. 2012;6:e1772.

46. Pratt-Riccio LR, Chehuan YF, Siqueira MJ, das Gracas Alecrim M, BiancoJunior C, Druilhe $\mathrm{P}$, et al. Use of a colorimetric (DELI) test for the evaluation of chemoresistance of Plasmodium falciparum and Plasmodium vivax to commonly used anti-plasmodial drugs in the Brazilian Amazon. Malar J. 2013;12:281.

47. Aguiar AC, Pereira DB, Amaral NS, De Marco L, Krettli AU. Plasmodium vivax and Plasmodium falciparum ex vivo susceptibility to anti-malarials and gene characterization in Rondonia, West Amazon, Brazil. Malar J. 2014;13:73.

48. Pava Z, Handayuni I, Wirjanata G, To S, Trianty L, Noviyanti R, et al. Expression of Plasmodium vivax crt-o is related to parasite stage but not ex vivo chloroquine susceptibility. Antimicrob Agents Chemother. 2015;60:361-7.

49. Wirjanata G, Sebayang BF, Chalfein F, Prayoga Handayuni I, Noviyanti R, et al. Contrasting ex vivo efficacies of "reversed chloroquine" compounds in chloroquine-resistant Plasmodium falciparum and P. vivax isolates. Antimicrob Agents Chemother. 2015;59:5721-6.

50. Rungsihirunrat K, Muhamad P, Chaijaroenkul W, Kuesap J, Na-Bangchang K. Plasmodium vivax drug resistance genes; Pvmdr1 and Pvcrt-o polymorphisms in relation to chloroquine sensitivity from a malaria endemic area of Thailand. Korean J Parasitol. 2015;53:43-9.

51. Sutanto I, Endawati D, Ling LH, Laihad F, Setiabudy R, Baird JK. Evaluation of chloroquine therapy for vivax and falciparum malaria in southern Sumatra, western Indonesia. Malar J. 2010;9:52

52. Grigg MJ, William T, Menon J, Barber BE, Wilkes CS, Rajahram GS, et al. Efficacy of Artesunate-mefloquine for chloroquine-resistant Plasmodium vivax malaria in Malaysia: an open-label, randomized, controlled trial. Clin Infect Dis. 2016;62:1403-11.

53. Siqueira AM, Alencar AC, Melo GC, Magalhaes BL, Machado K, Alencar Filho $A C$, et al. Fixed-dose artesunate-amodiaquine combination vs 
chloroquine for treatment of uncomplicated blood stage P. vivax infection in the Brazilian Amazon: an open-label randomized, controlled trial. Clin Infect Dis. 2017;64:166-74.

54. Nyunt MH, Han JH, Wang B, Aye KM, Aye KH, Lee SK, et al. Clinical and molecular surveillance of drug resistant vivax malaria in Myanmar (2009-2016). Malar J. 2017;16:117.

55. Singh L, Rathi S, Kondekar S, Kondekar A, Dongare S. Per protocol analysis of therapeutic efficacy of chloroquine for the treatment of Plasmodium vivax malaria in children. Int J Contemp Pediatr. 2016;3:725-9.

56. Seifu S, Zeynudin A, Zemene E, Suleman S, Biruksew A. Therapeutic efficacy of chloroquine for the treatment of Plasmodium vivax malaria among outpatients at Shawa Robit Health Care Centre, North-East Ethiopia. Acta Trop. 2017;171:44-51

57. World Health Organization. National treatment guidelines for malaria in Cambodia. Geneva: World Health Organization; 2012.

58. Hayward R, Saliba KJ, Kirk K. pfmdr1 mutations associated with chloroquine resistance incur a fitness cost in Plasmodium falciparum. Mol Microbiol. 2005;55:1285-95.

59. Henry M, Alibert S, Orlandi-Pradines E, Bogreau H, Fusai T, Rogier C, et al. Chloroquine resistance reversal agents as promising antimalarial drugs. Curr Drug Targets. 2006;7:935-48.

60. Melo GC, Monteiro WM, Siqueira AM, Silva SR, Magalhaes BM, Alencar $A C$, et al. Expression levels of pvcrt-o and pvmdr-1 are associated with chloroquine resistance and severe Plasmodium vivax malaria in patients of the Brazilian Amazon. PLoS ONE. 2014;9:e105922.

61. Barnadas C, Timinao L, Javati S, Iga J, Malau E, Koepfli C, et al. Significant geographical differences in prevalence of mutations associated with Plasmodium falciparum and Plasmodium vivax drug resistance in two regions from Papua New Guinea. Malar J. 2015;14:399.

62. Lu F, Lim CS, Nam DH, Kim K, Lin K, Kim TS, et al. Genetic polymorphism in pvmdr1 and pvcrt-o genes in relation to in vitro drug susceptibility of Plasmodium vivax isolates from malaria-endemic countries. Acta Trop. 2011;117:69-75.
63. Auburn S, Serre D, Pearson RD, Amato R, Sriprawat K, To S, et al. Genomic analysis reveals a common breakpoint in amplifications of the Plasmodium vivax multidrug resistance 1 locus in Thailand. J Infect Dis. 2016;214:1235-42.

64. Givens MB, Lin JT, Lon C, Gosi P, Char MC, Lanteri CA, et al. Development of a capillary electrophoresis-based heteroduplex tracking assay to measure in-host genetic diversity of initial and recurrent Plasmodium vivax infections in Cambodia. J Clin Microbiol. 2014;52:298-301.

65. Lin JT, Ubalee R, Lon C, Balasubramanian S, Kuntawunginn W, Rahman R, et al. Microscopic Plasmodium falciparum gametocytemia and infectivity to mosquitoes in Cambodia. J Infect Dis. 2016;213:1491-4.

66. Parobek CM, Lin JT, Saunders DL, Barnett EJ, Lon C, Lanteri CA, et al. Selective sweep suggests transcriptional regulation may underlie Plasmodium vivax resilience to malaria control measures in Cambodia. Proc Natl Acad Sci USA. 2016;113:E8096-105.

67. Costa GL, Amaral LC, Fontes CJF, Carvalho LH, de Brito CFA, de Sousa TN. Assessment of copy number variation in genes related to drug resistance in Plasmodium vivax and Plasmodium falciparum isolates from the Brazilian Amazon and a systematic review of the literature. Malar J. 2017;16:152.

68. Vargas-Rodriguez Rdel C, da Silva Bastos M, Menezes MJ, Orjuela-Sanchez P. Ferreira MU. Single-nucleotide polymorphism and copy number variation of the multidrug resistance-1 locus of Plasmodium vivax: local and global patterns. Am J Trop Med Hyg. 2012;87:813-21.

69. Woodrow CJ, Dahlstrom S, Cooksey R, Flegg JA, Le Nagard H, Mentre F, et al. High-throughput analysis of antimalarial susceptibility data by the WorldWide Antimalarial Resistance Network (WWARN) in vitro analysis and reporting tool. Antimicrob Agents Chemother. 2013;57:3121-30.

70. Woodrow CJ, Wangsing C, Sriprawat K, Christensen PR, Nosten F, Renia $L$, et al. Comparison between flow cytometry, microscopy, and lactate dehydrogenase-based enzyme-linked immunosorbent assay for Plasmodium falciparum drug susceptibility testing under field conditions. J Clin Microbiol. 2015;53:3296-303.

\section{Submit your next manuscript to BioMed Central and we will help you at every step:}

- We accept pre-submission inquiries

- Our selector tool helps you to find the most relevant journal

- We provide round the clock customer support

- Convenient online submission

- Thorough peer review

- Inclusion in PubMed and all major indexing services

- Maximum visibility for your research

Submit your manuscript at www.biomedcentral.com/submit
(O) BioMed Central 